\title{
Measurement of dC Vector Analyzing Power and Cross Sections at COSY for EDM Polarimetry
}

\author{
Fabian Müller for the JEDI Collaboration* \\ Forschungszentrum Jülich, IKP-2 | RWTH Aachen \\ E-mail: fa.muellerefz-juelich.de
}

\begin{abstract}
The JEDI (Jülich Electric Dipole moment Investigations) collaboration performs a set of experiments at the COSY storage ring of the Forschungszentrum Jülich in Germany, within the R\&D phase to search for the Electric Dipole Moments (EDMs) of charged particles. A measurement of proton and deuteron EDMs is a sensitive probe of yet unknown CP violation. An EDM observation would also be an indication for physics beyond the Standard Model.

The method of charged particle EDM search will exploit stored polarized beams in order to observe a miniscule rotation of the polarization axis as a function of time due to the interaction of a finite EDM with large electric fields. Key challenge is the provision of a sensitive and efficient method to determine the tiny change of the beam polarization. Elastic scattering of the beam particles on carbon nuclei will provide the polarimetry reaction. For this reason and as an input for future Monte-Carlo simulations, a good knowledge of the vector analyzing power and the unpolarized elastic cross section of deuterons scattered off a carbon target is very important to be able to determine the deuteron polarization. Over the course our experiment, the analyzing power and the differential cross section was measured using six different beam energies starting from $170 \mathrm{MeV}$ up to $380 \mathrm{MeV}$ deuterons.
\end{abstract}

23rd International Spin Physics Symposium - SPIN2018 -

10-14 September, 2018

Ferrara, Italy

* Speaker. 


\section{Introduction}

One of the great challenges in modern physics is to gain an understanding of the origin of the matter-antimatter imbalance observed in the universe. A necessary conditions for this disequilibrium is the violation of $\mathrm{CP}$ symmetry. The amount of $\mathrm{CP}$ violation predicted by the Standard Model of particle physics is orders of magnitude to low to explain this imbalance. A new source of $\mathrm{CP}$ violation is therefore needed and possible candidates are the Electric Dipole Moments (EDM) of elementary particles [1].

The general approach to measure EDM, is to observe the interaction of polarized particle with an electric field perpendicular to their spin axis. As the spin is the only quantization axis in a particle, the EDM has to be align to it. The electric field will therefore create a polarization buildup that can be measured using a polarimetry reaction. The predicted upper limit of $10^{-29} e \cdot \mathrm{cm}$ for a deuteron EDM is very small (the effect from the Magnetic Dipole Moment on the polarization is orders of magnitude larger) and hence a very precise polarimeter is needed to measure the miniscule change in polarization.

The polarimetry reaction intended to be used by the JEDI collaboration in their EDM experiment is elastic scattering of charged polarized particles such as protons and deuterons off an unpolarized carbon target. To obtain the polarization from this reaction, the knowledge of the vector analyzing power $A_{y}$ for the elastic scattering is of crucial importance. Together with the elastic cross section $d \sigma / d \Omega$, it will be used in the development of a designated polarimeter for the future EDM experiment performed by the members of the JEDI collaboration [2].

\section{The Experimental Setup}

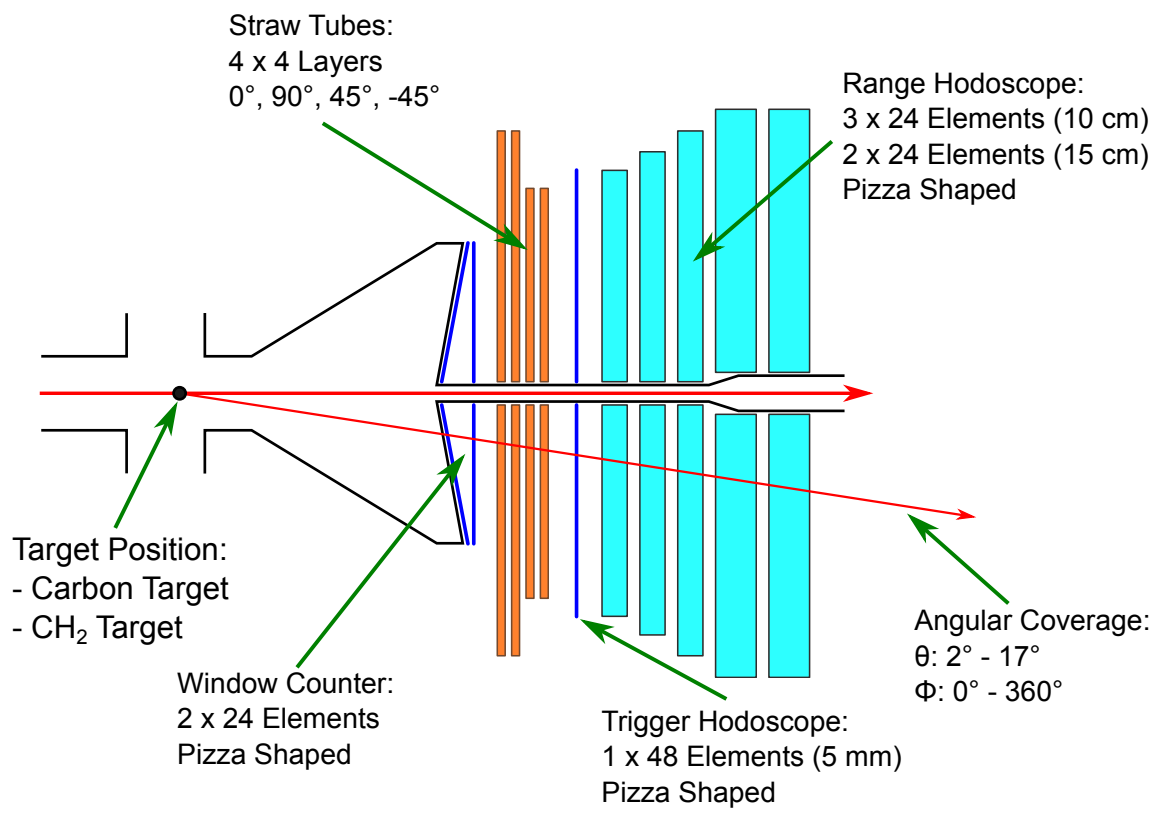

Figure 1: Schematic view of the WASA forward detector. 
The WASA Database Experiment was performed in November 2016 with the aim of measuring the vector analyzing power $A_{y}$ as well as the cross section $d \sigma / d \Omega$ for elastic deuteron scattering off a carbon target. The measurements were done using the WASA forward detector [3] (see Figure 1), installed at the COSY (cooler synchrotron) storage ring, as a polarimeter. The experiment used deuteron beams with seven different energies of $170 \mathrm{MeV}, 200 \mathrm{MeV}, 235 \mathrm{MeV}, 270 \mathrm{MeV}$, $300 \mathrm{MeV}, 340 \mathrm{MeV}$ and $380 \mathrm{MeV}$. Beams with two opposing vertically polarization as well as an unpolarized one were used for the measurement of elastic scattering off a thin diamond as well as off a Polyethylene $\left(\mathrm{CH}_{2}\right)$ target. The measured data was divided into polar angle $(\Theta)$ bins. For each $\Theta$-bin, two-dimensional histograms with the deposited energy in one layer of the detector versus the deposited energy of the subsequent layer were produced. From this spectra, the elastically scattered events could be selected using a graphical cut. This method was applied for the polarized data used in the asymmetry extraction as well to the unpolarized data used for the cross section calculation.

\section{Extracting the Vector Analyzing Power}

In order to measure the vector analyzing power, a quantity called the asymmetry $\varepsilon$ has to be measured. It describes the ratio of scattered events recorded in the left and right side of the detector respectively. The expected number of events is defined as follows:

$$
\left\langle N_{L, R}^{\uparrow, \downarrow}\right\rangle=\mathscr{L}^{\uparrow, \downarrow} \alpha_{L, R} \sigma_{0}(\Theta)(1+\varepsilon \cos (\Phi)) \text { with } \varepsilon=\frac{3}{2} A_{y}(\Theta) P_{y}
$$

Here, $\left\langle N_{L, R}^{\uparrow, \downarrow}\right\rangle$ denotes expected the number of events measured in the left $(\mathrm{L})$ and right $(\mathrm{R})$ side of the detector with a beam which is polarized upwards $(\uparrow)$ and downwards $(\downarrow)$. Further, $\mathscr{L}^{\uparrow, \downarrow}$ describes the luminosity, $\alpha_{L, R}$ the detector acceptance and $\sigma_{0}$ the unpolarized elastic cross section. The asymmetry is also defined as the product of the vector analyzing power $A_{y}$, vector polarization of the beam, $P_{y}$, and a spin- 1 specific factor of $3 / 2$.

For each polarization, the elastic events were integrated over the full azimuthal $(\Phi)$ range of the detector. If one just used event counts in the analysis, the usage of the full azimuthal range is not optimal in terms of statistical accuracy. Events close to $\Phi=\frac{\pi}{2}$ and $\frac{3}{2} \pi$ dilute the sample because $\cos (\Phi)=0$. For this reason, a weight was assigned to each event in the following way:

$$
m_{n}^{p}=\sum_{i}^{e v(p)} \cos \left(\Phi_{i}\right)^{n} \text { for } n=0,1,2
$$

With $p \in[\uparrow, \downarrow, 0]$ describing the polarization states up, down and unpolarized. Note, that $m_{0}^{p}$ simply corresponds to the sum of the events. $m_{1}^{p}$ is the weighted sum, where events at $\Phi=\frac{\pi}{2}$ and $\frac{3}{2} \pi$ are suppressed because their weight is zero. From this weighted sums, the asymmetries for the upwards and downward polarized beam could be calculated using the method of Extraction of azimuthal asymmetries using optimal observables described in [4].

As the magnitude of the polarization of the beam $P_{y}$ was unknown, the asymmetry for $200 \mathrm{MeV}$ and $270 \mathrm{MeV}$ was fitted to published reference vector analyzing powers ([5] and [6]) to obtain the beam polarization values. The line shape of the measured asymmetry is in good agreement with the published data (see Figure $2 \mathrm{a}$ ). The polarization obtained using this method varied by $\sim 15 \%$ 
which lead to rather large systematic uncertainties (red regions in Figure 2a). The polarization value was used to calculate $A_{y}$ for all seven beam energies and is shown in Figure $2 \mathrm{~b}$.

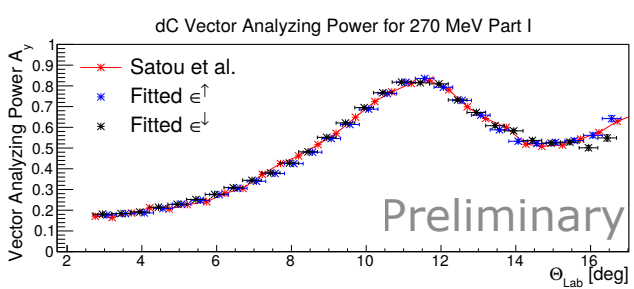

dC Vector Analyzing Power for $270 \mathrm{MeV}$ Part II

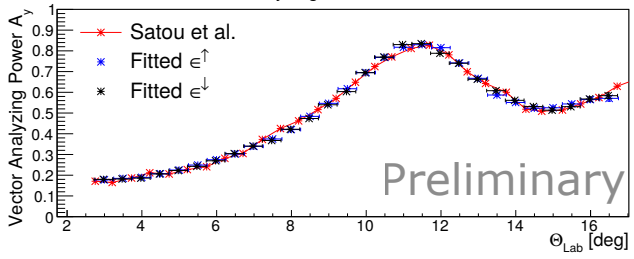

dC Vector Analyzing Power for $200 \mathrm{MeV}$

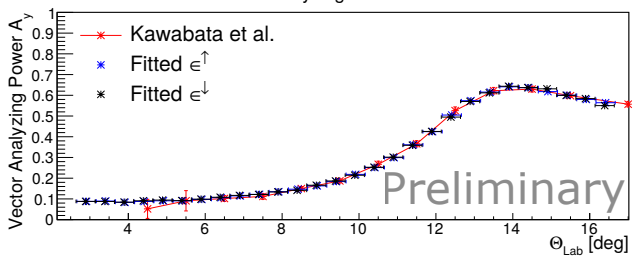

(a) Measured asymmetry for the upwards $\varepsilon^{\uparrow}$ (blue) and downwards $\varepsilon^{\downarrow}$ (black) polarized beam. The asymmetries were fitted to the reference data (red) by Satou et al. and Kawabata et al. to obtain the beam polarization. The $270 \mathrm{MeV}$ data was measured in two parts that were fitted individually.
Vector Analyzing Power for Elastic dC Scattering

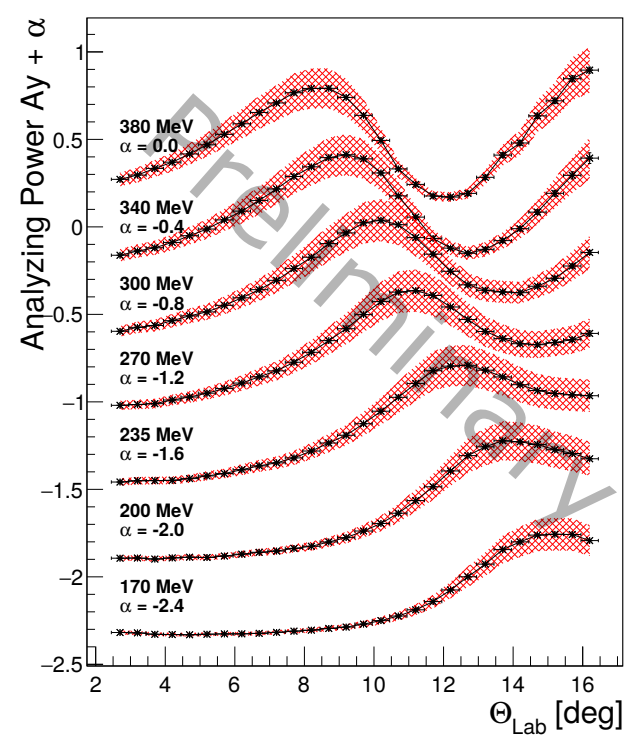

(b) Reconstructed vector analyzing power for the deuteron beam energies of (from top to bottom) $380 \mathrm{MeV}, 340 \mathrm{MeV}, 300 \mathrm{MeV}, 270 \mathrm{MeV}, 235 \mathrm{MeV}$, $200 \mathrm{MeV}$ and $170 \mathrm{MeV}$. The curves are subsequently offset by 0.4 for better readability. The statistical errors are indicated with the black error bars on the data points. The systematic error is draw as the red regions.

Figure 2: Results of the vector analyzing power extraction.

\section{Extraction of the Unpolarized Cross Section}

The elastic event selection procedure for the data was applied to a Monte-Carlo simulation. From the result, the ratio between generated and accepted events was calculated leading to the detector acceptance for each beam energy.

To calculate the cross section, the luminosity is needed but since the WASA detector was installed on the beam-pipe of COSY, the flux could not be measured directly because a flux detector would have been to destructive to the beam in the storage ring. To overcome this issue, the elastically scattered deuteron proton events off the hydrogen in the polyethylene target were extracted as well. The acceptance for the deuteron proton scattering was again calculated from Monte-Carlo. By fitting the acceptance-corrected deuteron proton spectrum to published reference data for the elastic proton deuteron cross sections, the luminosity for this reaction was obtained. The flux of the beam particle is not reaction dependent and thus the luminosity for the deuteron carbon scattering could be calculated from the deuteron proton scattering. 
This method of normalizing the acceptance-corrected deuteron carbon data resulted in cross sections that are in agreement with published cross section by Kawabata et al. for $200 \mathrm{MeV}[5]$ and by Satou et al. for $270 \mathrm{MeV}$ [6] at the $10 \%$ level as can be seen in Figure 3b. The results for all seven energies are given in Figure 3a.

\section{Elastic dC Cross Sections}

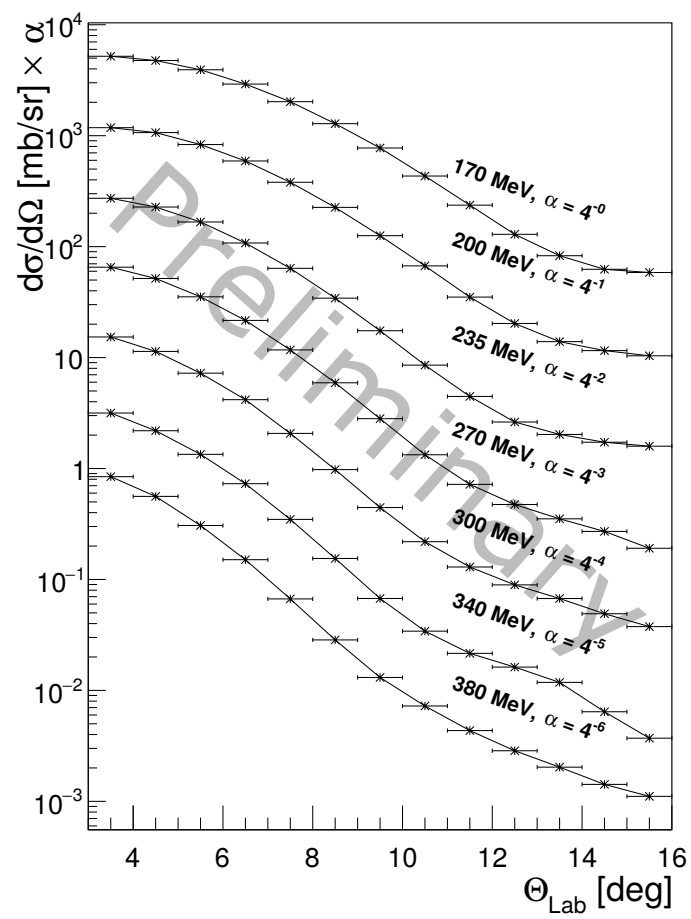

(a) Elastic deuteron carbon cross sections for the energies of (from top to bottom) $170 \mathrm{MeV}, 200 \mathrm{MeV}, 235 \mathrm{MeV}$, $270 \mathrm{MeV}, 300 \mathrm{MeV}, 340 \mathrm{MeV}$ and $380 \mathrm{MeV}$. The curves are subsequently scaled down by a factor of 4 for better readability.
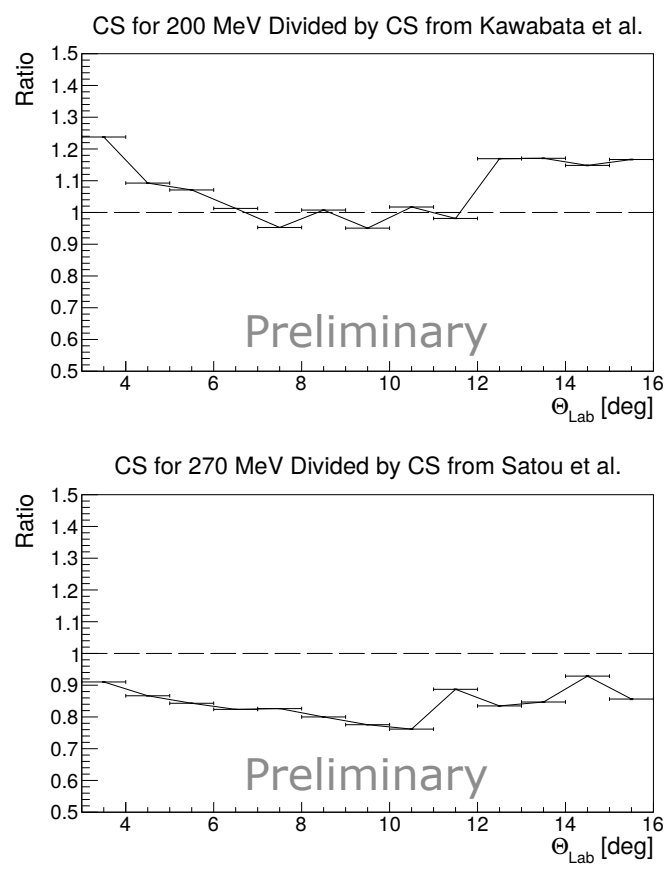

(b) Comparison of the cross section from this work and the published ones by Kawabata et al. for $200 \mathrm{MeV}$ (top) and Satou et al. for $270 \mathrm{MeV}$ (bottom) by calculating their ratio.

Figure 3: Results of the elastic deuteron carbon differential cross section.

\section{Figure of Merit of the Polarization}

The statistical error on the EDM scales with the statistical error of the vector polarization $\Delta P_{y}$. The goal is thus to minimize this quantity. In order to use the results shown above to optimize a designated polarimeter, it is convenient to define a Figure of Merit (FoM) as follows:

$$
\mathrm{FoM}=\frac{\langle\omega \beta\rangle^{2}}{\left\langle\omega^{2}\right\rangle} \sigma \sim \frac{1}{\left(\Delta P_{y}\right)^{2}}
$$

with $\langle\beta\rangle=\frac{\int_{\Delta \Omega(\Delta \Theta, \Delta \Phi)} \beta(\Theta, \Phi)\left(\frac{d \sigma}{d \Omega}\right) d \Omega}{\int_{\Delta \Omega(\Delta \Theta, \Delta \Phi)}\left(\frac{d \sigma}{d \Omega}\right) d \Omega}$ and $\sigma=\int_{\Delta \Omega(\Delta \Theta, \Delta \Phi)}\left(\frac{d \sigma}{d \Omega}\right) d \Omega$ 
With $\beta(\Theta, \Phi)=\frac{3}{2} A_{y}(\Theta) \cos (\Phi)$ as defined in Equation (3.1) and $\omega$ an arbitrary weight. By maximizing the FoM, one minimizes the statistical error on the vector polarization of the beam. In [7], it is shown that the statistical limit is reached by choosing $\omega=\beta(\Theta, \Phi)=\frac{3}{2} A_{y}(\Theta) \cos (\Phi)$. This also allows to use the full azimuthal range, i.e. $\Delta \Phi=2 \pi$, for the calculation of the FoM. Equation (5.1) can thus be rewritten as:

$$
\mathrm{FoM}=\left(\frac{3}{2}\right)^{2} \pi \int^{\Delta \Theta} A_{y}(\Theta)^{2} \sin (\Theta)\left(\frac{d \sigma}{d \Omega}\right) d \Theta .
$$

The actual value of for the FoM depends on the polar integration range $\Delta \Theta$ as well as on the beam energy. Figure 4 provides an overview of the FoM depending on these two quantities.

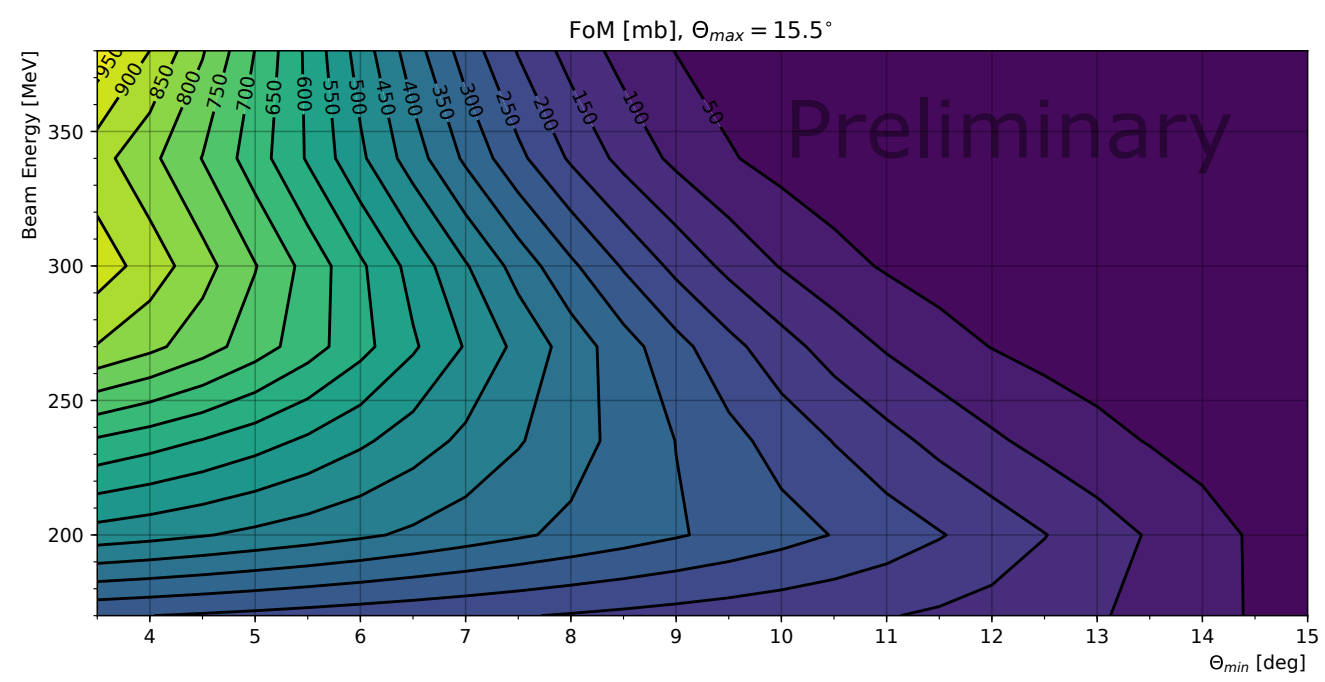

Figure 4: Figure of Merit as a function of the beam energy and the integration range. The integration range is defined as $\Delta \Theta=\left[\Theta_{\min }, \Theta_{\max }\right]$. The upper limit is fixed to $\Theta_{\max }=15.5^{\circ}$ while the lower limit is given on the $\mathrm{X}$-axis. From this map it is possible to find either the best integration range for a given beam energy or to find the best beam energy for a given integration range (i.e. angular coverage of the detector).

\section{Summary and Conclusion}

Measuring an EDM of charged particle in a storage ring demands on a very precise polarization build-up determination. A designated polarimeter is under development by members of the JEDI collaboration [8]. Optimizing this device and performing high accuracy analysis of the miniscule rotation of the polarization axis requires a good knowledge of the vector analyzing power and cross section of the reaction of interest, which in this case is elastically deuteron carbon scattering. The analysis of the data measured by the WASA detector produced results for the vector analyzing power $A_{y}$ as well as the unpolarized cross section $d \sigma / d \Omega$ for seven beam energies of $170 \mathrm{MeV}$ up to $380 \mathrm{MeV}$. Combining these two quantities allowed to create a map for the Figure of Merit indicating the ideal angular coverage range and beam energy for a future polarimeter from a statistical point of view. 
The results for $A_{y}$ presented here are in good agreement with published data concerning their line shape. The lack of direct measurements of the beam polarization meant that the measured asymmetries had to be fitted to published reference data to obtain the polarization. Unfortunately, the results from these fits introduced a large systematic uncertainty in the result of $A_{y}$.

The extraction of the unpolarized elastic deuteron carbon cross section produced results that are comparable with published data. Despite the rather complicated method of calculating the luminosity from a second reaction, it was possible to produce an acceptable result.

The Figure of Merit can be used to determine ideal parameters for a designated polarimeter but it is important to keep in mind that other arguments than pure statistics have to be taken into account when designing this device.

\section{Acknowledgements}

This work has been financially supported by the European Research Council Advanced-Grant (srEDM, No. 694340). The author wants to express his gratitude to Edward Stephenson and Volker Hejny and especially to Maria Żurek for her guidance and support and for the huge amount of work she has put into the preparation and analysis of the data.

\section{References}

[1] J. Engel, M. J. Ramsey-Musolf and U. van Kolck, Electric Dipole Moments of Nucleons, Nuclei, and Atoms:The Standard Model and Beyond, Prog. Part. Nucl. Phys. 7121 (2013)

[2] JEDI Collaboration, http://collaborations.fz-juelich.de/ikp/jedi/

[3] H.H. Adamet al.(WASA-at-COSY), proposal for the wide angle shower apparatus(wasa) at cosy-julich, Wasa at cosy (2004), nucl-ex/ 0411038

[4] J. Pretz and F. Müller, Extraction of azimuthal asymmetries using optimal observables, The European Physical Journal C 79:47 (2019)

[5] T. Kawabata et al, Isovector and isoscalar spin-flip M1 strengths in ${ }^{11}$ B, Physical Review C 70 (2004) 034318

[6] Y. Satou et al, Three-body dN interaction in the analysis of the ${ }^{12} C\left(\vec{d}, d^{\prime}\right)$ reaction at $270 \mathrm{MeV}$, Physics Letters B 549 (2002) 307-313

[7] J. Pretz, Comparison of methods to extract an asymmetry parameter from data, Nuclear Instruments and Methods A659 (2011) 456-461

[8] F. Müller, The EDM polarimeter development at COSY-Jülich, Hyperfine Interactions 10 (2019) 240 